\title{
A Systematic Review: The Association Between Social Media Use, Social Media Addiction, and Depression in Adolescents
}

\author{
Letong Yang ${ }^{1, *}, \dagger$, Jingqiao Yuan ${ }^{2, \dagger}$ \\ ${ }^{1}$ Psychology department, University of Washington, Seattle, 98105, U.S.A. \\ ${ }^{2}$ Zhou Enlai School of Government, Nankai University, Tianjin, 300350, CHINA. \\ ${ }^{*}$ Corresponding author. Email: letong@uw.edu
}

These authors contributed equally.

\begin{abstract}
With the development of technology, social media has become the main way for people to communicate with others and entertain themselves. At the same time, depression is one of the most common mental disorders. About $4.3 \%$ of people in the world are diagnosed with depression. Many researchers pay attention to the relationship between social media and depression. They did surveys to measure depression and the level of social media addiction or use and tested the correlation between them. Surveys are only able to get a correlation but not accurate causation, and most related researches used the same method. Because of simply using surveys during research, mechanisms between social media use, social media addiction, and depression need further exploration. Our review aims at finding the universal result of various previous researches and presenting suggestions on method selection for later researches. We reviewed 15 articles and offered a summary of previous methods and results. Our review found that the methods used are limited, and it is necessary to find new approaches to get a more specific relationship. Controversial results suggested that further and more careful research are needed. A universally used scale for depression and social media use or addiction would be helpful for investigating related fields. They should also focus on the mechanism but not only the correlation between some variables.
\end{abstract}

Keywords: Social media, social media use, social media addiction, depression, internet addiction, Facebook addiction, young adults, adolescents

\section{INTRODUCTION}

Social media is an internet-based media, and it provides an opportunity to build quick electronic communication of content [1]. Multiple platforms are designed for different purposes of social media interaction. Some of the platforms are mainly designed for direct one-to-one communication or interaction (i.e., WhatsApp). Others' targeting customers are those who engage with community sharing (i.e., Instagram and Twitter). According to Pew Research Center, at least $92 \%$ of young adults in the US visit social media at least once a day [2]. With the increasing use of social media, more researches focus on topics related to social media use. In fact, social media has always been a double-edged sword. On the one hand, engagement on social media may increase social capital through more social interactions [3]. On the other hand, critiques on social media suggested that social media may cause stigmatizing mental health problems [4, 5]. Twenge pointed out that young people engaged with social media overuse are more likely to do harm to social development and interaction [6]. Researchers kept questioning whether excessive use of social media may eventually turn into potential addictive behavior. According to Andereassen and Pallesen, addictive social media use was defined as "being overly concerned about social media, driven by an uncontrollable motivation to $\log$ on to or use social media, and devoting so much time and effort to social media that it impairs other important life areas [4]." Recent studies have focused on how daily social media use is related to social media addiction on its compulsiveness and loss of self-control [7]. The relationship between social media use and social media 
addiction is worth investigating. However, before research on the association between social media use and social media addiction, two variables must be differentiated clearly and precisely.

Daily use of social media, especially when it is excessive, has been long obscured with problematic social media use [8]. Lee et al. conducted a study to investigate the psychiatric comorbidity differences between daily social media users and addictive social media users [9]. A previous study also identified that there was a significant difference in the six core criteria of addiction (e.g., Salence, mood modification, tolerance, withdrawal, conflict, and relapse) [10]. In the previous studies that discussed the relationship between daily social media use and addictive use, researchers found that daily social media use showed a positively correlated pattern with social media addiction, especially when focused on the social media usage frequency $[5,7]$. Griffith et al. further clearly distinguished the differences between daily overuse and problematic use [10]. Generally, social media use was mostly able to predict social media addiction.

Depression was one of the major mental health disorders according to DSM-V [11]. Depressive symptoms consist of depressed mood, loss of interest, weight gain or loss, insomnia or hypersomnia, and etc. As one of the major mental health problems, depression causes patients to suffer from impairment in daily activities such as studying or working. For severe depression, it may even lead to suicide. An early study [12] showed that social media addiction predicted high estimates of depressive symptoms. A recent study emphasized that problematic social media use was positively associated with depression [13]. Nevertheless, there were also opposing results put forward by Andreassen et al. that problematic social media use was associated negatively with a decrease in depressive symptoms due to higher social interaction even though it's online and decreases loneliness which is a symptom of depression [4]. Much of the multifaced findings on the association between depression and problematic social media use brings a thorough identification. The present review includes multiple research studies aimed at integrating findings and results on correlations between social media use, social media addiction, and depression. This review also includes covariates and how they influence the former variables.

\section{METHODOLOGY}

Eligible studies A comprehensive literature search was conducted with the following search and extraction criteria: (1) The databases Google Scholar, PubMed, Elsevier, HathiTrust were used. (2). Keywords of Social media, social media use, social media addiction, depression, mental illness, internet addiction, Facebook addiction, young adults and adolescents are included for article selection. (3) Eligible studies with a targeting population excluding the age of under 12. (4) Excluded were those measuring smartphone addiction and depression since smartphones have multiple uses other than social media usage and might bring bias to the review. (5) Social media were defined as websites for social interaction, including Facebook, Instagram, Twitter, WhatsApp, and etc. (6) All articles were in the English language. And one study measuring Internet Addiction relates to depression is also included. Finally, 15 articles were included in our review.

\subsection{Methods of Previous Researches}

In the 17 articles we reviewed, 15 of them used the survey to collect data. The participants of these studies included adults, university students and adolescents [7] $[14,15]$. The main method of these studies was measuring depression and social media addiction using scales. The scales they used to measure depression are different, including the Depression Anxiety Stress Scale , Beck Depression Inventory, Center for Epidemiological Studies Depression Scale for Children [15-17]. The scale they used to measure social media addiction includes: Social Network Addiction Questionnaire, Generalized Problematic Internet Use Scale 2 and Internet Addiction Proneness Scale for Youth-Short From [5, 15, 18].

The other two articles used the corpus to do linguistic analysis [19, 20]. It was a new way to detect the relationship between social media addiction and depression. They built a linguistically annotated corpus with the sentiment analysis, and they aimed to study young people behavior through their social media message and also to find out the relationship between the message that people sent and depression. In the studies using sentiment analysis, researchers also used some natural language processing tools and applied machine learning methods to predict behavior. In other words, these studies took social media as a platform to collect data and connect it with depression symptoms.

The method used now is limited, so later researches can expand in the methodology, like making a combination of survey and linguistic analysis or using some experiments to get a more concrete relationship rather than just a correlation.

\subsection{Basic Information of 15 Studies}

Of the 15 studies, 12 were correlational, and 3 were longitudinal. The total sample across the studies was 36768 individuals and 423 families. Six studies are conducted in the United States, three in Turkey, and one each in Bangladesh, Afghanistan, Norway, Korea, Spain, and Finland. Most studies focused solely on social media use, social media addiction and depression. One study from Bangladeshi focused on Facebook addiction alone, whereas three studies from Turkey, Korea and a 
longitudinal study focused on Internet addiction rather than specifically on social media addiction $[15,18,21$, 22]. In the research from the US, social media use was measured, and the study did not mention the concept of social media addiction [23]. Two studies clarified the social media use separately with time spent and frequency [7, 24]. Except for the investigation of the social media influence on depression, three studies measured covariates, i.e., self-esteem, loneliness, and suicidal ideation. Besides, multiple studies covered exposure to confounding variables, including age, race, gender, education level, and other socio-demographics.

\subsection{Social Media Use and Addiction}

Within 12 cross-sectional studies, four studies distinguished the concept of social media use and social media addiction as two distinct variables. These four papers have also investigated the relationship between daily social media use and social media addiction. Studies collected daily social media use through surveys with Likert-scales or questionnaires. Some studies calculated and summed the daily reported hours into weekly use standards. For instance, researchers asked participants to report both daily use estimates and the social media use frequency for further analysis [7]. However, measuring social media addiction appeared to include multiple scales for evaluation. Despite the difficulty in consisting of the measuring scales, among four papers analysing the correlation between social media use and social media addiction, all found that there was a significant positive association [7]. For an article, which divided social media use in frequency and time, also found that both two divisions are significantly correlated to social media addiction. That is, longer time spent and higher frequency on social media is related to the higher probability of addictive behavior on social media. It is proper to say that there was a moderately consistent analysis of the relationship between social media use and social media addiction. Under various research methods, social media use and addiction presented a positively correlated relationship. On the concept definition, researchers either consider social media addiction as a maladaptive subtype of social media use or as a distinct behavior.

\subsection{Social Media Use Relates to Depression}

Putting forward onto depression, different measuring scales have been used. Despite the potential bias due to inconsistent measuring scales, the results from six studies measuring the correlation between social media use and depression showed an obvious pattern. In the articles, researchers analysed social media time spent and social media usage frequency, respectively, with depression [5, 7]. Both these two studies suggested that the amount of time spent on social media has no significant correlation with depression. Nevertheless, when investigated how social media usage frequency affected depression, they provided different results $[5,7]$. The variable they measured was social media use and depression. Three supported the idea that social media use and depression are significantly correlated. The results supporting the positive correlation consistent with the findings of previous studies and contradicted with Neira and Barber $[25,26]$. Except for the consistent pattern itself, those studies found no correlation pointed to an undeclared investigation. For an article, the results found that both daily social media use hours and depression were positively related to addictive behavior [5]. It is very likely that addictive social media use was an intermediate variable that connected social media use and depression. Some result suggested that social media use could improve depressive symptoms in Latino groups with acculturative stress [16]. It is reasonable to speculate that stressed group can gain improvement on social media use rather than experiencing negative outcome that leads to depression. The target groups and sample conditions are dissimilar to other studies. In short, social media use and depression in most studies are positively and significantly correlated.

\subsection{Social Media Addiction Relates to Depression}

After a systematic review of 15 studies included, social media addiction was found to be positively associated with depression in most of the studies. But there are also a few contradictory results on this relationship. Within all of the studies, nine of them tried to figure out the relationship between addictive behavior and depression. Of these researches, six examined the relationship between social media addiction and depression. Five of the six studies determined that social media addiction has a significant correlation with depression or depressive symptoms [4, 5, 7, 14, 24]. The one study left, however, presented a contradicting result [27]. Despite the fact that social media addiction has a positive correlation with depression, social media addiction and depression are related indirectly. Instead, the study suggested that social media addiction causes the time spent on social media to increase and thus trigger a higher possibility for depressive symptoms[27]. Internet addiction has been studied by two research groups $[15$, 22]. Instead of the specific concept of social media addiction, two studies turned out that Internet addiction also has a significantly positive association with depression $[15,22]$. Both studies that took place in Turkey and Korea shared the same result, and this consequence would need further exploration in different cultures. For the cross-sectional study of Manun \& Griffiths, the relationship between social media addiction and depression were explored in Facebook, and consistent with previous studies, the results showed that Facebook addiction was positively correlated with depression [21]. 


\subsection{Demographic Perspective}

Researchers measured some covariable like races, income, education level, socio-demographic information, gender, age, multiple social media, level of acculturation, connection with others and so on [28]. The results presented that people with younger age, being female, have lower education level, and not in a relationship are at more risk of social media addiction [5, 7]. Studies also supported that people with younger age, lower education level, lower income, and being single are also at more risk of depression [24, 29]. According to multiple studies, the at-risk groups for social media addiction and depression are largely overlapped [4, 5, 7, 14]. Although many results proved that girls who are depressed have a higher risk to be diagnosed with social media addiction, an article stated that there is no significant difference in correlation between social media addiction and depression among genders [18]. In an article, it is proved that highly acculturated Latinos shared a higher tendency to social media use and mental health problems [16]. An article also mentioned that multiracial groups are more related to depression [7]. These results lead to speculation that whether the effect of social media addiction and depression act more aggressively on multiracial groups and showed the many impact variables for social media addiction and depression. The implication of the articles studied needs more relevant studies to investigate the moderating effects, especially within subgroups under different social conditions. So later researches can focus on other variables which haven't been studied yet, including social media overuse, childhood experience with social media addiction, education background with depression and so on.

\section{DISCUSSION}

It should be highlighted that the scales they used for depression and social media addiction are both different because they should fit the sample they chose. It should have a universally used scale, especially for social media addiction, to confirm the validity. As for the linguistic analysis, it just focused on the message and the depression. It is a big leap for traditional researches about social media and depression. Actually, social media platforms have the most accurate information about people's use of social media. They know exactly how many times you use social media, what kind of video you like to watch, how much time you spend on social media every day. If we can make a scale for social media addiction using this information as measuring items, the result will be more accurate. And the scale can take Bergen Facebook Addiction Scale and Social Networking Website Addiction Scale as reference.

We can see that the method used for social media and depression research are very limited, and the correlation between social media addiction and depression are even sometimes not that valid. We should retest the result of previous researches. More importantly, we should find new methods. We can find more theoretical support or supply the methodology, and in operation, we can design some experiments. Making social media use as an independent variable and depression level as a dependent variable. Obviously, it is difficult as we should solve the moral problem and control many other variables. And for linguistic analysis, it is used to detect depression and avoid danger. And we can get more data from the social media company about people's use of social media and then connect them with depression symptoms so that we can find out the true causal relationship and use corresponding therapy.

\subsection{Difference in Various Social Media Platforms}

YouTube is used to watch some videos and upload your video, and Facebook and Twitter are used to chat with your friends. These are two main social media activities. If you are addicted to videos on social media, you may be attracted by views and scenes in the video, and you may be trapped in the virtual world. But in chatting social media, you may like to chat with others in a special way that is different from face-to-face communication. This kind of chatting may destroy your social ability, but it also proved beneficial in some studies. This kind of change may have an influence on your depression. Actually, many people use many social media at the same time, and this kind of using also associated with depression. It showed that the more social media platform you use, the higher odds of depression level. There aren't any research study the depression with a social media platform. Maybe some kinds of social media like chatting social media are easier to get people addicted to, and maybe video social media are associated with more serious depression. These are also the blank area that needs to study.

\subsection{Directionality of Results}

Within the 15 studies, those studies focused on the relationship between social media use and depression and found that higher social media use increases depressive symptom occurrence. This result is consistent with the relevant study of systematic reviews investigating social media use and depression. Similar results within dissimilar socio-demographic contents provide evidence that the research findings explain relationships regardless of geographical location, economic level, and political stability [30]. Various targeting groups with different age range also suggest that the relationship between social media use and depression are still mostly positive and significant.

In various studies, some consider social media addiction as a subtype of social media use with negative outcomes, whereas others consider social media 
addiction as another variable independent of social media use. In Jasso-Medrano \& López-Rosales (2018) 's study, the results attributed to the speculated model indicating that higher daily social media use experienced a higher possibility of social media addiction is related to depression.

Young first reported that the improvement of depression level has a connection with internet addiction, and after that, many researchers reported that social media use would increase the risk of depression [31]. Girls who have depression have a higher risk to be diagnosed with internet addiction. The mechanism between social media addiction and depression is unclear by now. There are two kinds of possible explanations about that: First, social media can give people a kind of social support, and it is easier to control the social activity, some that social media use can be a tool to release depression mood, so people use social media are more likely to addictive about social media [32]. Secondly, those who are addicted to social media, are lack social connections in the real world, so they need more social support, and they are easier to be depressed [33]. And most present researches are cross-sectional studies, so they can't get accurate causation. Some longitudinal studies get the opposite result. Some find social media use is the reason, and some think depression is the reason. A few researches also focused on intervening variables like sleeping quality. These researches aimed at finding the mechanism about how social media use affect depression. It gave us a new way to find out more reasons and helped us give more specific therapy. It mentioned that the using behavior didn't lead to depression directly but through some other intervening variable, and sleeping quality is one of them. Others like social activity quality and social support or some cognitive change may also be the intervening variable, and it needs further study as related studies are rare.

\section{CONCLUSION}

Current studies included cross-sectional studies and longitudinal studies. Both used questionnaires and surveys for information collection. Social media use is often self-reported with open-ended questions and sometimes collected through the filled Likert-scale. Depression symptoms or depressive levels are directly measured by valid scales, i.e., PROMIS, CES-D, BDI, etc. Nevertheless, self-report is possible to meet social desirability bias. To eliminate such biases, other research methods that are valid and suitable for the research are worthy of note. Although it takes a high amount of time, energy, and money spent, using social platforms to monitor usage with participants' permission might be a better way to measure social media use.

Since the investigation on how social media addiction act between social media use and depression are not included in the review, our implication is that addictive social media use as a mediator between social media use and depression. Not much related studies investigating social media addiction as a mediating role has been done. Future study should focus more on how addictive behavior act between social media use and depression.

\section{REFERENCES}

[1] Gosling, S. D., Augustine, A. A., Vazire, S., Holtzman, N., \& Gaddis, S. (2011). Mani- festations of personality in online social networks: Selfreported Facebook- related behaviors and observable profile information. Cyberpsychology, Behavior, and Social Networking, 14(9), 483e488.

[2] Pew Research Center. (2015). Social media update 2015. Retrieved June 8, 2016, from: http://www.webcitation.org/6ajEhvS11.

[3] de la Pena, A., \& Quintanilla, C. (2015). Share, like and achieve: The power of Facebook to reach health-related goals. International Journal of Consumer Studies, 39(5), 495e505. ELEC.

[4] Andreassen, C. S., Billieux, J., Griffiths, M. D., Kuss, D. J., Demetrovics, Z., Mazzoni, E., \& Pallesen, S. (2016). The relationship between addictive use of social media and video games and symptoms of psychiatric disorders: A large-scale cross-sectional study. Psychology of Addictive Behaviors, 30(2), 252-262. https://doi.org/10.1037/adb0000160.

[5] Jasso-Medrano, J. L., \& López-Rosales, F. (2018). Measuring the relationship between social media use and addictive behavior and depression and suicide ideation among university students. Computers in Human Behavior, 87, 183-191. https://doi.org/10.1016/j.chb.2018.05.003.

[6] Twenge, J. (2006). Generation me: Why we expect more from technology and less from each other. New York: Basic Books. Vandervoort, D. (1999). Quality of social support in mental and physical health. Current Psychology, 18(2), 205. Retrieved from:

https://link.springer.com/content/pdf/10.1007\%2Fs 12144-999-1029-8.pdf.

[7] Shensa, A., Escobar-Viera, C. G., Sidani, J. E., Bowman, N. D., Marshal, M. P., \& Primack, B. A. (2017). Problematic social media use and depressive symptoms among U.S. YOUNG adults: A NATIONALLY-REPRESENTATIVE STUDY. Social Science \& Medicine, 182, 150-157. doi:10.1016/j.socscimed.2017.03.061.

[8] Ryan, T., Chester, A., Reece, J., Xenos, S., The uses and abuses of Facebook: A review of Facebook addiction. Journal of Behavioral Addictions. 2014; 
3(3):133-48. doi: 10.1556/JBA.3.2014.016 PMID: 25317337.

[9] Lee, J., Park, E., Kwon, M., Choi, J., Jeong, J., Choi, J., . . Kim, D. (2014). The difference in comorbidities and behavioral aspects between Internet abuse and Internet dependence in Korean male adolescents. Psychiatry Investigation, 11(4), 387. doi:10.4306/pi.2014.11.4.387.

[10] D Griffiths, M. (2013). Social networking addiction: Emerging themes and issues. Journal of Addiction Research \& Therapy, 04(05). doi:10.4172/2155$6105.1000 \mathrm{e} 118$.

[11] Tolentino, J. C., \& Schmidt, S. L. (2018). DSM-5 Criteria and Depression Severity: Implications for Clinical Practice. Frontiers in psychiatry, 9, 450. https://doi.org/10.3389/fpsyt.2018.00450.

[12] Costello, E. J., Mustillo, S., Erkanli, A., Keeler, G., \& Angold, A. (2003). Prevalence and development of psychiatric disorders in childhood and adolescence. Archives of General Psychiatry, 60, 837-844.

[13] Donnelly, E., \& Kuss, D. J. (2016). Depression among users of social networking sites (SNSs): The role of SNS addiction and increased usage. Journal of Addiction and Preventive Medicine, 1(2), 107. Retrieved March 11, 2018 from http://irep.ntu.ac. uk/id/eprint/29245/.

[14] Haand, R., \& Shuwang, Z. (2020). The relationship between social media addiction and depression: A quantitative study among university students in KHOST, AFGHANISTAN. International Journal of Adolescence and Youth, 25(1), 780-786. https://doi.org/10.1080/02673843.2020.1741407.

[15] Park, S., Hong, K. M., Park, E. J., Ha, K. S., \& Yoo, H. J. (2012). The Association between problematic internet use and depression, suicidal ideation and bipolar disorder symptoms in Korean adolescents. Australian \& New Zealand Journal of Psychiatry, 47(2), 153-159. doi:10.1177/0004867412463613.

[16] Franco, J. A., \& Carrier, L. M. (2020). Social media use and depression, anxiety, and stress IN Latinos: A correlational study. Human Behavior and Emerging Technologies, 2(3), 227-241. doi:10.1002/hbe2.205.

[17] Coyne, S. M., Rogers, A. A., Zurcher, J. D., Stockdale, L., \& Booth, M. (2020). Does time spent using social media impact Mental Health?: An eight year longitudinal study. Computers in Human Behavior, 104, 106160. doi:10.1016/j.chb.2019.106160.
[18] Gámez-Guadix, M. (2014). Depressive symptoms and problematic internet use among adolescents: Analysis of the longitudinal relationships from the cognitive-behavioral model. Cyberpsychology, Behavior, and Social Networking, 17(11), 714-719. doi:10.1089/cyber.2014.0226.

[19] Li, A., Jiao, D., \& Zhu, T. (2018). Detecting depression stigma on social media: A linguistic analysis. Journal of Affective Disorders(), doi:10.1016/j.jad.2018.02.087.

[20] Zaghouani, W. (2018).A Large-Scale Social Media Corpus for the Detection of Youth Depression (Project Note). Procedia Computer Science, doi:10.1016/j.procs.2018.10.483.

[21] Mamun, M. A., \& Griffiths, M. D. (2019). The Association between Facebook addiction and Depression: A pilot survey study AMONG Bangladeshi students. Psychiatry Research, 271, 628-633. doi:10.1016/j.psychres.2018.12.039.

[22] Demir, Y., \& Kutlu, M. (2016). The relationship between loneliness and depression: Mediation role of internet addiction. Educational Process: International Journal, 5(2), 97-105. https://doi.org/10.12973/edupij.2016.52.1.

[23] Lin, L. Y., Sidani, J. E., Shensa, A., Radovic, A., Miller, E., Colditz, J. B., . . . Primack, B. A. (2016). ASSOCIATION between social media use and depression Among U.S. young adults. Depression and Anxiety, 33(4), 323-331. doi:10.1002/da.22466.

[24] Aydin, S., Koçak, O., Shaw, T. A., Buber, B., Akpinar, E. Z., \& Younis, M. Z. (2021). Investigation of the effect of social media addiction on adults with depression. Healthcare, 9(4), 450. https://doi.org/10.3390/healthcare9040450.

[25] Tiggemann, M., \& Slater, A. (2015). The role of self-objectification in the mental health of early adolescent girls: Predictors and consequences. Journal of Pediatric Psychology, 40, 704-711.

[26] Neira, C.J.B., \& Barber, B. L. (2014). Social networking site use: linked to adolescents' social self-concept, self-esteem, and depressed mood. Australian Journal of Psychology, 66, 56-64.

[27] Kircaburun, K. (2016). Self-Esteem, Daily Internet Use and Social Media Addiction as Predictors of Depression among Turkish Adolescents. Journal of Education and Practice, 7(24), 64-72.

[28] Primack, B. A., Shensa, A., Escobar-Viera, C. G., Barrett, E. L., Sidani, J. E., Colditz, J. B., \& James, A. E. (2017). Use of multiple social media platforms and symptoms of depression and anxiety: A NATIONALLY-REPRESENTATIVE study among 
U.S. young adults. Computers in Human Behavior, 69, 1-9. doi:10.1016/j.chb.2016.11.013.

[29] Puukko, K., Hietajärvi, L., Maksniemi, E., Alho, K., \& Salmela-Aro, K. (2020). Social media use and DEPRESSIVE symptoms - a longitudinal study from early to late adolescence. International Journal of Environmental Research and Public Health, 17(16), 5921. doi:10.3390/ijerph17165921.

[30] Keles, B., McCrae, N., \& Grealish, A. (2019). A systematic review: The influence of social media on depression, anxiety and psychological distress in adolescents. International Journal of Adolescence and Youth, 25(1), 79-93. https://doi.org/10.1080/02673843.2019.1590851.

[31] Young, K. S., Rogers, R. C.. (1998). The relationship between depression and Internet addiction. Cyberpsychology \& behavior, 1(1), 2528.

[32] Ko, C. H., Yen, J. Y., Chen, C. S., Yeh, Y. C., \& Yen, C. F. (2009). Predictive values of psychiatric symptoms for internet addiction in adolescents: a 2year prospective study. Archives of pediatrics \& adolescent medicine, 163(10), 937-943.

[33] Kraut, R., Patterson, M., Lundmark, V., Kiesler, S., Mukophadhyay, T., \& Scherlis, W. (1998). Internet paradox: A social technology that reduces social involvement and psychological well-being?. American psychologist, 53(9), 1017. 